$<$ VERSO> R. Durón Figueroa et al.

$<$ RECTO $>$ Early Intervention for ASD Earthquake Victims

\title{
$<$ AT > Efficacy of an Early Cognitive-Behavioral Intervention for Acute Stress Disorder in Mexican Earthquake Victims
}

<AU> Raúl Durón-Figueroa ${ }^{1}$, Georgina Cárdenas-López ${ }^{1}$, and Soledad Quero²

<AFF1> Universidad Autónoma Nacional de México (Mexico)

<AFF2> Universitat Jaume I (Spain)

\section{Conflicts of Interest: None}

Funding Statement: Supported by the National Council of Science and Technology

(CONACyT) graduate scholarship program

<ACK> National Autonomous University of Mexico (UNAM) and National Council of Science and Technology (CONACyT)

$<\mathrm{COR}>$ Correspondence concerning this article should be addressed to Raúl Durón Figueroa. Universidad Autónoma Nacional de México. 04510 Coyoacan (Mexico). E-mail: raulduron7@gmail.com

How to cite this article:

Durón-Figueroa, R., Cárdenas-López, G., \& Quero, S. (2020). Efficacy of an early cognitivebehavioral intervention for acute stress disorder in Mexican earthquake victims. The Spanish Journal of Psychology, 23. EXX. http://doi.org/XXX
Comentat [u1]: Hay que poner un guión entre los dos apellidos???

Comentat [u2]: Hay que poner un guión entre los dos apellidos??? 


\begin{abstract}
$<\mathrm{ABS}>$ Abstract
Acute stress disorder (ASD) refers to the symptoms associated with posttraumatic stress disorder (PTSD) within the first four weeks following the traumatic event. Recent theoretical models suggest that early detection of ASD provides an opportunity to implement early interventions to prevent the development of PTSD or ameliorate its symptomatology. The aim of the present study was the evaluation of the efficacy of an ASD treatment for earthquake victims, which would serve as an early intervention for PTSD. A single-case $(n=1)$ quasi-experimental design was used, with pre and post-assessments, as well as one, three and six-month follow-ups, with direct treatment replications. Fourteen participants completed the treatment and the follow-up measurements. The results obtained using a single-case analysis showed significant clinical improvement and clinically significant change when employing a clinical significance analysis and the reliable index of change. Statistical analyses of the dataset displayed statistically significant differences between the pre and post-assessments and the follow-up measures, as well as large effect sizes in all clinical measures. These results suggest that the treatment was an efficacious early intervention for PTSD during the months following the traumatic event, although some relevant study limitations are discussed in the text.
\end{abstract}

$<$ HIS> Received 17 February 2020; Revised XXXXXX; Accepted XXXXXX.

$<$ KWD> Keywords: acute stress disorder, early intervention, posttraumatic stress disorder, trauma. 
Exposure to traumatic events such as violent episodes or natural disasters increases the risk of significant deterioration of mental health, which might lead to anxiety, depression, substance abuse, as well as the development of more serious trauma and stressor-related disorders, such as acute stress disorder (ASD) and posttraumatic stress disorder (PTSD) (Norris et al., 2002).

More specifically, diverse psychological consequences have been found in people who have experienced natural disasters, such as symptoms of anxiety and depression, as well as symptoms linked to trauma-related disorders. An example of this is a systematic review (Galea et al., 2005), which found a 5 to $60 \%$ prevalence of PTSD between the first and second year following the traumatic event. Consistent with this, another systematic review which included 116 studies analyzing the development of PTSD related to natural disasters (Neria et al., 2008) revealed a prevalence of the disorder ranging from 3.7 to $60 \%$. It is important to note that some reviews have found that earthquakes and tsunamis are the most common triggers for the development of PTSD symptoms (Cova \& Rincón, 2010). Considering the duration of ASD and its relationship to PTSD, there are few specific epidemiological studies regarding earthquakes. However, some studies have found a high rate of ASD and subsequent PTSD symptoms later months (Sattler et al., 2006; Soldatos et al., 2006)

Despite a scarcity of studies including the Mexican population, some relevant data have been collected regarding natural disasters and PTSD. One of the most notorious disasters in the country's history was the 1985 Mexico City earthquake, and a $32 \%$ PTSD prevalence was identified in a sample of 524 participants (Tapia et al., 1987). A $2.3 \%$ PTSD prevalence has been found as a consequence of natural disasters (Medina-Mora et al., 2005), and $3.6 \%$ of male and $2.6 \%$ of female adolescents ( 12 to 17 years of age) that have been victims of natural disasters exhibit PTSD symptoms (Orozco et al., 2008). Speaking specifically of the 2017 earthquake in Mexico, some studies have been carried out where it has been found that a prevalence of PTSD of $34.6 \%$ occurred (Abeldaño et al., 2019). In summary, there is significant evidence that natural disasters, mainly earthquakes, result in the development of PTSD as one of the most serious sequels of traumatic events.

One of the most salient issues regarding the recent study of trauma and stressor-related disorders has been the relationship between ASD and PTSD. ASD was recognized by the DSM-IV (American Psychiatric Association; APA, 1994) in order to describe the symptoms associated to PTSD within the first four weeks following the event, since during this time lapse it is possible to identify the typical symptoms of reliving the event, avoidance and activation, as well as dissociation; thus, this disorder has been an object of study regarding its relation to 
and its level of prediction of PTSD (Mordeno \& Cue, 2015). Even though recent studies and reviews have provided a strong case for a significant prediction level, it has been found that there is a lack of sufficient empirical evidence to consider ASD as a robust predictor of PTSD (Bryant, 2018). However, given the recent modifications implemented in the DSM-5 (APA, 2013), it has been found that giving less weight to dissociative symptoms in the diagnosis of ASD results in a stronger prediction of PTSD (Bryant et al., 2010; Bryant et al., 2012; Bryant et al., 2015). It is important to note that the previously mentioned changes were not explicitly directed towards increasing the prediction power of ASD, but merely intended to optimize the diagnosis of the disorder, as well as to promote early interventions for PTSD (Bryant, 2018). In this sense, it has been found that cognitive-behavioral therapy (CBT) using prolonged exposure (PE) has been particularly effective in the treatment of ASD and PTSD, becoming the first line of treatment for both (Bryant, 2016). This intervention focuses on the exposure of the client to traumatic memories, as well as related situations, in order to achieve an adequate emotional processing (McLean \& Foa, 2011). In general, it has been found that the implementation of PE CBT at the initial stages of trauma-related disorder symptoms has yielded effective results in the prevention of PTSD during the following months (Kliem \& Kröger, 2013).

As a result, different studies have been carried out regarding early interventions to prevent the development of PTSD. A systematic review (Birur et al., 2017) including 58 studies focused on early interventions to prevent the onset of PTSD found that psychological treatments, especially PE CBT, implemented during the acute phase following the trauma were effective in avoiding the development of PTSD during the first weeks following the event. Even though not all studies took into account an ASD diagnosis, the majority identified PTSD symptoms during the initial weeks, which would imply a possible ASD presence. Added to this, another systematic review (Howlett \& Stein, 2016) revealed that the majority of studies focused on successful early PE CBT PTSD prevention, implemented during the first weeks following the trauma were directed toward an ASD population or, at the very least, samples exhibiting ASD symptoms.

Given the psychosocial relevance of trauma and stressor-related disorders, as well as the existence of novel theoretical approaches and the need of implementing early PTSD interventions such as the one proposed in DSM-5 (APA, 2013), it is important to carry out new studies based on diagnostic and conceptual modifications 
and recommendations stemming from updated reviews regarding the pertinent constructs (Bryant, 2018). The earthquake that took place in Mexico City on September 19, 2017, was one of the worst natural disasters in recent years, and required a prompt response to meet the mental health needs of the affected population. Considering that the protocol used had been previously adapted for victims of violence, the protocol was modified in terms of the materials to be used, considering them in situations of natural disasters, directing the clinical intervention of this project towards the victims who presented the symptoms of such disorders as a result of the earthquake.

Consequently, the present study aimed to evaluate the efficacy of a CBT program through PE for ASD in Mexican earthquakes victims, which was proposed as an early intervention in the prevention of PTSD.

\section{$<\mathrm{H} 1>$ Method}

\section{$<\mathrm{H} 2>$ Participants}

Following the earthquake that took place in Mexico City on September 19, 2017, the participants were referred by health organizations and community attention brigades of a Mexican university. Initially, 40 individuals, who requested emotional support through the centers mentioned, were assessed using the Mexican version of the Acute Stress Disorder Scale (Bryant et al., 2000; Durón et al., 2018), which was employed in order to establish an initial diagnosis and, using the recommended cutoff point (> 56), to identify persons at risk of developing PTSD. From the 40 assessed participants, 25 met the criteria for ASD, and 14 agreed to take part in the intervention program. This group consisted of 12 women $(85.7 \%)$ and two men $(14.3 \%)$, between 22 and 70 years of age, with a mean of $40.71(S D=15.44)$. Regarding their civil status, eight were single (57.1\%), four were married (28.6\%), one was divorced $(7.1 \%)$, and one was widowed $(7.1 \%)$. In terms of education, one had completed primary school (7.1\%), five had a high school education (35.7\%), and eight possessed a higher education $(57.1 \%)$. Regarding the degree of exposure to the event, the participants were in the workplace or at home during the earthquake, however, none were found in situations of greater danger, such as trapped in fallen buildings or collapses. All 14 participants completed the treatment program, as well as the post-assessment and one, three and six-month follow-ups.

\section{$<\mathrm{H} 2>$ Design}

In order to assess the treatment program efficacy, a single-case quasi-experimental design was used, with pre and post-assessments, as well as one, three and six-month followups, with direct treatment replications (Barlow \& Hersen, 1984; Kazdin, 2011).
Comentat [SJP3]: Tengan en cuenta la siguiente observación del editor asociado: "No se hace referencia en el texto a que el estudio haya recibido la aprobación de un comité ético. Esto ya fue señalado en una primera revisión. En su respuesta, los autores obvian esta cuestión, aunque sí explicitan que cuentan con un consentimiento informado. Se

ruega que se aclare este punto".
POR FAVOR, MARQUEN EN OTRO COLOR EN EL TEXTO EL LUGAR DÓNDE REALIZAN TAL ACLARACIÓN 


\section{$<\mathrm{H} 2>$ Instruments}

Acute Stress Disorder Scale (ASDS; Bryant et al., 2000). This scale is based on the Acute Stress Disorder Structured Interview (Bryant et al., 1998). It has 19 items that describe the ASD symptomatology, and scored with a Likert scale ranging from 1 (not at all) to 5 (very much). Regarding the score, the authors propose a cutoff point of $>56$, which serves to identify the individuals at risk of developing PTSD. The adapted scale for Mexico has an appropriate internal consistency index (.96), as well as an adequate convergent validity ( $r s=.61$ to .88 ) and factorial adjustment (Durón-Figueroa et al., 2018).

PTSD Checklist for DSM-5 (PCL-5; Weathers et al., 2013). The instrument is based on the PTSD Checklist (PCL; Weathers, 2008), and consists of 20 items graded using a Likert scale from 0 (not at all) to 4 (extremely). The authors suggest a score higher than 33 in order to obtain a partial PTSD diagnosis. The Mexican version possesses a high internal consistency index (.96), appropriate convergent validity ( $r s=$ .58 to .88 ) and factorial adjustment (Durón-Figueroa et al., 2019).

Beck Anxiety Inventory (BAI; Beck et al., 1988). The inventory has 21 items that determine the severity of symptomatic and behave oral categories. Its psychometric properties in the Mexican population (Robles et al., 2001) show a high internal consistency (.84 and .83$)$ and test-retest reliability $(r=.75)$.

Beck Depression Inventory (BDI; Beck et al., 1961). The BDI consists of two factors comprising 21 items. It evaluates affective/cognitive and vegetative/somatic symptoms using a Likert scale with values ranging from 0 (no depressive symptom) to 3 (severe symptom). The Mexican version has a Cronbach's alpha of .94 (Jurado et al., 1998).

Maladjustment Scale (MS; Echeburúa et al., 2000). This scale consists of six items which assess the degree of maladjustment to different daily life situations as a consequence of specific problems. It uses a scale that goes from 0 (not at all) to 5 (a lot), and has a Cronbach's alpha of .94 .

\section{$<\mathrm{H} 2>$ Procedure}

Between the first and second week following the earthquake, individuals interested in taking part in the program were contacted via telephone by the therapists, and the ASDS was administered, identifying the DSM-5 diagnostic criteria (APA, 2013) for their inclusion in the study. Despite the fact that the scale was designed under the DSM-IV criteria, the authors consider that it can be used with the DSM-5 criteria 
(APA, 2013), taking as reference the presence of 9 of the intrusive symptoms that the diagnosis describes (Bryant, 2013). Although the participants began to ask for help in the first week, it was decided to carry out the evaluation between the first and the second week, since it has been recommended to wait and identify if the symptoms are persistent (Bryant, 2016). As part of the inclusion criteria, in addition to the diagnostic criteria of the DSM-5, participants over 18 years old and who accepted informed consent were considered; in turn, it was considered as exclusion criteria that they were receiving some other psychological treatment.

Taking into account the social problems and the emotional consequences that resulted from the earthquake, all persons who did not meet the inclusion criteria where referred to alternate emotional support programs. Subsequently, the selected participants took part in an admission interview in order to explore in detail the trauma-related symptomatology, and completed the additional assessment instruments. This second specific interview was conducted in the spaces where the treatment was carried out. Despite not being subject to an ethical committee review, informed consent was used from the study, which was responded in this evaluation session.

After the conclusion of the treatment, a post-assessment was carried out, and one, three and six-month follow-up evaluations were scheduled. Considering the temporary nature of ASD and its relation to PTSD symptoms, the PCL-5 was administered in order to compare the pre-assessment measures with the results following the intervention and to evaluate the extent to which the intervention program served as a preventive measure for the development of PTSD. Additionally, the anxiety and depression inventories were used, as well as the maladjustment scale.

\section{$<$ REFJ> Treatment}

The treatment protocol used is based on the Mexican adaptation of the "Acute stress disorder treatment guidelines" (Bryant, 2007). The treatment consists of an individual cognitive-behavioral program of 6-8 weekly sessions, each lasting approximately 90 minutes. The first session of the program focuses on explaining the basis of the intervention, the techniques to be employed, and psychoeducation concerning the common symptoms and reactions following a traumatic event. Training in slow diaphragmatic breathing as an anxietycoping measure is introduced, and homework is given, based on the techniques reviewed during the session. The second session includes the explanation of the ABC model as a part of the cognitive therapy for handling automatic negative thoughts related to the traumatic event. Also, the rationale for the PE therapy using imagination is introduced and the first exposure is carried out. During the exposure, the therapists keep a record of anxiety levels using the subjective 
units of distress (SUDs), which are registered before the exposure, every five minutes during the exposure, and after its conclusion. The third session focuses on explaining in vivo exposure, which is used as a part of PE during the homework assignments. Once this technique is described, a hierarchy of stressful situations is created, which will have to be covered between sessions, with the first assignment given during this session. Additionally, cognitive therapy focused on negative thoughts and exposure therapy using imagination continue to be used. The fourth and fifth sessions continue the exposure and cognitive therapy, and the progress regarding the hierarchical in vivo exposure is examined, with the assignment of pertinent homework. Finally, the sixth session covers the review of the in vivo exposure according to the progress through the hierarchy scale, the last exposure using imagination, and the cognitive components. Furthermore, a relapse prevention is included, as well as the scheduling of follow-up sessions. It is important to mention that all the participants completed the five exposure sessions established in the protocol. Likewise, three expert therapists in anxiety disorders and trauma-related disorders participated, who carried out the specific protocol mentioned and, in turn, weekly supervision sessions were held to verify that they were carrying out the sessions in accordance with the protocol.

\section{$<\mathrm{REFJ}>$ Data Analysis}

Considering the research design used in the present study, a case-by-case analysis was first undertaken through visual inspection, clinical significance and the reliable change index (RCI; Jacobson \& Truax, 1991) for each participant. As part of the clinical significance analysis and the RCI, the methods proposed by Jacobson and Truax (1991) were used, in which a low cutoff point (Criterion A) was established, where the sum of the mean and two standard deviations of the dysfunctional population are calculated. In cases where clinical improvement is seen in a decrease of the scale values, as was the case in this study, the value of the standard deviations is inverted to the negative. On the other hand, the second condition of the employed method is the RCI, which assesses the degree of clinically significant change with the pre and postassessment, based on the reliability degree of each instrument. In order to consider a treatment as clinically significant, the established cutoff point must be surpassed and the indices determined by the RCI must be reached. Owing to this, the authors propose four categories in relation to the levels of change: (a) recovered, when the cutoff point and the RCI are reached; (b) improved, when only the RCI is reached; (c) no change, 
when the RCI is not reached, regardless of the cutoff point; and (d) deteriorated, when an RCI in the opposite direction of the functionality values is present.

Additionally, statistical analyses were carried out for the dataset to compare the pre and post-treatment measures, as well as the follow-up results. For this, Wilcoxon rank-sum test. Finally, the effect size was calculated using Cohen's $d$, and the calculations were corrected with Hedge's $g$, which is specifically recommended for small samples $(<20)$ (Cumming, 2012).

\section{$<\mathrm{H} 1>$ Results \\ $<\mathrm{H} 2>$ Sample Characteristics}

Concerning clinical characteristics, all participants met the DSM-5 diagnostic criteria for ASD (APA, 2013) when assessed between the first and second week following the traumatic event. A mean of $66(S D=7.55)$ was found using the ASDS, with a range of 57-80, a score above 56 being the cutoff point to identify ASD, as well as a risk of developing PTSD during the following months. The PCL -5 , which uses a cutoff score of 33 for meeting the diagnostic criteria, showed a mean of $46.21(S D=9.13)$, with a 38-73 range. In terms of associated symptomatology, the results of the BAI indicated a moderate to severe index of anxiety symptoms with a mean of $26.43(S D=9.41)$ and scores ranging from 16 to 52 , whereas the BDI resulted in a mean of $15.86(S D=5.09)$, with a range between 10 and 26, interpreted as a mild to moderate depression. Lastly, the $M S$ revealed a mean of $16.43(S D=4.21)$ and a range of 12-25, 12 being the minimal score to indicate a significant maladjustment degree.

\section{$<\mathrm{H} 2>$ Case by Case Analysis \\ $<\mathrm{H} 3>$ Visual Inspection and Clinical Improvement}

Final scores and cutoff points of the assessment instruments were used to carry out the visual inspection and clinical improvement analyses of each participant. For this purpose, patterns of change were analyzed using individual participant graphs in order to observe possible tendencies and, in turn, the reduction degree of the symptomatology was analyzed by means of the scores obtained on each scale and diagnostic criteria; the graphs used for the visual inspection can be seen in the Appendix. Apart from considering the obtained scores and cutoff points, a percentage change (or reduction) of the different clinical measures was undertaken based on the scores and the visual inspection; a recommended reference for determining whether a clinically significant change has taken place would be a minimal reduction of $50 \%$ in the severity and frequency of the symptoms (Blanchard \& Schwartz, 1988). 
Based on the mentioned method, considering the PTSD scale, the initial range was between 38 and 73, with 33 being the cutoff score for a PTSD diagnosis. Afterwards, all participants had scores below this cutoff point at post-assessment and follow-up measures. According to the scores obtained during post-assessment, there was a decrease in symptoms between 67 and 97\%, 71 and 100\% at the one-month follow-up, 79 and $97 \%$ at the three-month follow-up, and 77 and $100 \%$ at the six-month follow-up. Furthermore, visual inspection showed a similar tendency among all participants of a significant decrease between the pre and post-assessment, as well as a stability or maintenance of the improvement regarding the post-assessment and the follow-up measures.

As mentioned earlier, the initial BAI scores had a range between 16 and 52, indicating moderate to severe anxiety levels. During post-assessment, scores between 3 and 13 were observed, with a decrease in symptomatology between 50 and $89 \%$. Regarding the follow-up measures, scores between 1 and 11 with a decrease of 55-97\% were present at the one-month follow-up, scores ranging from 0 to 9 with a decrease of $50-97 \%$ were seen at the three-month follow-up, as well as scores between 0 and 9 with a decrease of $62-100 \%$ at the six-month follow-up. Likewise, visual inspection showed a tendency similar to the PTSD measures, with a significant reduction of symptoms at post-assessment and a maintenance of improvement during follow-ups.

In the case of depression symptoms, similar clinical improvements were found. Initially, scores between 10 and 26 were present, showing mild to moderate depression levels. Taking into account the fact that some participants displayed low depression levels during pre-assessment, they exhibited no significant improvement, which was corroborated by visual inspection and change percentages following treatment. During post-assessment, scores between 0 and 10, and a decrease between 25 and $90 \%$ were found. At the one-month follow-up assessment, scores ranging from 0 to 10 with a 16 $100 \%$ decrease were observed. At the three-month follow-up, scores between 0 and 9 with a $25-100 \%$ reduction were seen. Lastly, a score range of $0-8$ and a $33-100 \%$ decrease were present at the six-month follow-up. Excluding the participants that showed low depression scores during the initial evaluation, visual inspection yielded results in reduction and maintenance similar to the previous measures.

Finally, the $M S$ showed a range between 12 and 22 points, all these scores being consistent with a significant maladjustment. During post-assessment, the participants showed scores between 0 and 6, with a 66-100\% reduction. Score ranges of 0-5 (66- 
100\% decrease), 0-5 (66-100\% decrease) and 0-4 (86-100\% decrease) were detected at one, three and six-month follow-ups, respectively. Similar to the previous results, visual inspection confirmed a significant improvement and maintenance of the changes.

\section{$<\mathrm{H} 3>$ Clinical Significance and RCI}

In accordance with the analyses performed, and the categories proposed by Jacobson and Truax (1991), Table 1 displays the results obtained for the 14 participants. In the case of PTSD measures, it can be observed that all participants could be classified as "recovered" at post-assessment and follow-up measures.

In terms of clinical measures of anxiety, half of participants could be considered as "recovered" at post-assessment, whereas four would be categorized as "improved", and 3 as "no change". At the one-month follow-up, the majority of participants could be categorized as "recovered", with the rest being classified as "improved". Three months after the intervention, most participants would belong to the "recovered" category, four participants to the "improved" category, and one could be classified as "no change". At the six-month follow-up, 13 participants belonged to the "recovered" category, with only one being considered "improved".

Regarding depression measures, the majority of participants were considered as "recovered" at post-assessment, five participants were "improved", and one participant was "no change". At the one-month follow-up, most participants were considered "recovered", two were "improved", and one was "unchanged". After three months, 10 participants were classified as "recovered", three were placed in the "improved" category, and one was "no change". At the six-month follow-up, 11 participants were considered "recovered", and the rest belonged to the "improved" category.

Finally, concerning the measures of maladjustment and interference, all participants were considered "recovered" at the post-assessment and subsequent follow-ups.

\section{<Insert Table 1 Here>}

\section{$<\mathrm{H} 2>$ Intra-group Comparison Statistical Analyses}

As seen in Table 2, the results obtained with the Wilcoxon test showed statistically significant differences in all clinical measures $(p<.001)$ between the pre and post-assessments, as well as one, three and six-month follow-ups. Additionally, the calculation of the effect size, considering the cutoff point of .80 for a large effect (Cohen, 1988), found large effect sizes in all clinical measures in the post-assessment and all subsequent follow-ups.

\section{<Insert Table 2 Here>}




\section{$<\mathrm{H} 1>$ Discussion}

The aim of the present study was to evaluate the efficacy of a CBT using PE for ASD in the victims of the Mexico City earthquake that took place on September 19, 2017. In addition, it was expected that this treatment would serve as an early intervention for the prevention of PTSD during the following months.

According to recent studies and theoretical innovations about trauma and stressor-related disorders, as well as new diagnostic criteria of the DSM-5 (APA, 2013), it has become easier to identify people with ASD who are thus at risk of developing PTSD during the first weeks following the traumatic event. Consequently, the implementation of early interventions during those initial weeks might prevent a possible onset of PTSD.

The results of the study showed a significant reduction in symptoms of traumarelated disorders, which is consistent with the empirical evidence regarding the use of PE for these disorders (Bryant, 2016; McLean \& Foa, 2011). Generally, it is possible to observe a significant clinical improvement between the pre and post-assessment, in addition to the measurements of one, three and six-month follow-ups. These results indicate an absence of symptomatology of PTSD, pointing toward the efficacy of the proposed early intervention, which is consistent with the study proposals and previous reviews (Bryant et al., 2015; Bryant, 2018). Furthermore, clinically significant improvements regarding the additional measures of anxiety, depression and maladjustment were also observed after the intervention, as well as the subsequent follow-ups. It is important to mention that applying a single case methodology is important for several reasons such as the fact that this type of design can contribute to research on the efficacy of psychological interventions and shows that this is a research that could be applied in a simple way in the applied practice of clinical psychology, in order to detect if the psychological intervention applied with a particular person or persons generates a reliable and clinically significant change.

Concerning the case-by-case analysis, the results showed clinical improvements in all the participants that completed the treatment. Using the different clinical measures, the visual inspection revealed a similar pattern across all cases, where initial elevated scores decreased after treatment and the improvement was maintained during follow-ups. When analyzing the different scores obtained through multiple measurement scales, as well as the diagnostic criteria, an absence of PTSD symptoms 
was seen, in addition to a significant reduction in anxiety, depression and maladjustment symptoms. Considering the study design employed, and the replications of the treatment in different participants with their respective outcomes, a generalization due to treatment can be inferred (Kazdin, 2011). Although the visual inspection method has turned out to be common in this type of study, it is important to mention that it can present various biases, so special care must be taken in the interpretation of results (Campbell \& Herzinger, 2010).

The results obtained from the clinical significance analyses and RCI also showed clinically significant changes in all participants for the different clinical variables employed, according to the criteria set by Jacobson and Truax (1991). Regarding the PTSD measures, it was observed that $100 \%$ of participants were classified as "recovered" at the post-assessment and the follow-ups, which indicates an absence of PTSD in the months following the treatment. In turn, the anxiety measures showed that the majority of participants belonged to the "recovered" and "improved" categories, with the exception of three participants at postassessment and one participant during follow-up, who could be classified as "no change". Similarly, the depression measures indicated that more than $90 \%$ of the participants were classified as "recovered" and "improved", excluding one participant who remained "no change" at post-assessment and follow-ups. Finally, regarding the maladjustment measures, all participants were found to be "recovered" at different points of assessment after the intervention.

It is important to mention that, as was the case of anxiety and depression measures in this study, some authors mention that the RCI might be affected when the obtained scores at pre-assessment are close to those of the functional population (Iraurgi, 2010). In the present study, both measures exhibited low and moderate scores when initially evaluated and, in spite of showing a clear improvement, did not meet the standards of the employed method, resulting in some participants being classified as "no change". However, it is worth repeating that neither of these measures displayed elevated scores after treatment. In conclusion, the analyses suggest that the intervention of the present study resulted in clinically significant changes for all participants in the different clinical variables.

Apart from the case-by-case examination, statistical analyses were carried out in order to compare the means of the dataset. In general, these analyses showed statistically significant differences in all the clinical variables between the pre and post-assessments, in addition to the one, three and six-month follow-ups. Moreover, the calculation of effect sizes showed large sizes for all the clinical measures. These results are consistent with the case-by-case analysis and clinical significance, providing additional evidence of treatment efficacy. 
The present results are consistent with previous studies, which suggest that CBT using PE reduces the symptoms of trauma-related disorders through an emotional processing of traumatic memories (Foa \& Kozak, 1986; McLean \& Foa, 2011). In the specific case of ASD, the results also point towards the usefulness of this type of early interventions in the prevention of PTSD (Bryant et al., 2015). Furthermore, when taking into account the significant discomfort and interference caused by associated symptoms, as well as the subsequent maladjustment to the traumatic event (APA, 2000), the results of this study suggest that the program was efficacious in the treatment of these variables, achieving a positive readjustment to the day-to-day activities that were disrupted in the aftermath of the earthquake.

Despite the fact that the results are promising for the treatment of ASD and the prevention of PTSD, the present study possesses certain limitations that are worth considering. As has been reported in the relevant literature (Bryant, 2016), one of the most important constraints when studying ASD is the short time span of the disorder, and this significantly limits the ability to conduct controlled studies. In the present case, the use of repeated measures for the baseline would have made a more rigorous control possible; however, considering the characteristics of the disorder, as well as the serious social implications of this particular disaster, such methodological elements were deemed unsuitable. Another important limitation of the study was the reduced sample size. Although the design used allows the generalization of results via treatment replication with different participants, it is important to have a larger number of cases in order to obtain results that represent the general population. Also, it is important to undertake studies with different types of trauma in order to generalize the results, while this study focused solely on the effects of one specific earthquake.

With these limitations in mind, further studies with a more strict methodological control and a more representative sample of traumatic experiences are recommended. Given the recent theoretical updates and studies regarding trauma-related disorders, more research about this topic will be able to enrich the literature about efficacious treatments and the implementation of early interventions for individuals at risk of developing PTSD.

In general, the present study provided evidence for the efficacy of CBT using PE for ASD in earthquake victims, as well as the prevention of PTSD in the subsequent months. Considering the fact that ASD is a scarcely studied topic, this work represents a contribution to the recent literature regarding the disorder. Nevertheless, it is 
important to consider the limitations of the study, as well as the recommendations for future research, which could provide a more solid empirical support for these findings. 


\section{$<\mathrm{H} 1>$ References}

<REFJ> Abeldaño Zuñiga, R., Guerrero Reyes, G., Siliceo Murrieta, J., \& González Villoria, R. (2019). Posttraumatic stress symptoms in people exposed to the 2017 earthquakes in Mexico. Psychiatry Research, 275, 326-331. https://doi.org/10.1016/j.psychres.2019.04.003

$<$ REFJ> American Psychiatric Association (1994). Diagnostic and statistical manual of mental disorders (4 $4^{\text {th }}$ Ed.). Author.

$<$ REFJ $>$ American Psychiatric Association (2000). Diagnostic and statistical manual of mental disorders (4th ed.). Washington, DC: Author.

$<$ REFJ $>$ American Psychiatric Association (2013). Diagnostic and statistical manual of mental disorders (5 $5^{\text {th }}$ Ed.). Author. https://doi.org/10.1176/appi.books.9780890425596

$<$ REFJ> Barlow, D., \& Hersen, M. (1984). Single case experimental designs. Strategies for studying behavior change $\left(2^{\text {nd }}\right.$ Ed.). Pergamon Press.

$<$ REFJ>Beck, A., Epstein, N., Brown, G. y Steer, R. (1988). An inventory for measuring clinical anxiety: Psychometric properties. Journal of Consulting and Clinical Psychology, 56 (6), 893 897. https://doi.org/10.1037/0022-006x.56.6.893

<REFJ>Beck, A., Ward, CH., Mendelson, M., Mock, J. y Erbaugh, J. (1961). An inventory for measuring depression. Archives of General Psychiatry, 4, 561-571. https://doi.org/10.1001/archpsyc.1961.01710120031004

$<$ REFJ > Blanchard, E. B., \& Schwarz, S. P. (1988). Clinically significant changes in behavioral medicine. Behavioral Assessment, 10, 171-188.

<REFJ> Birur, B., Moore, N. C., \& Davis, L. L. (2017). An evidence-based review of early intervention and prevention of posttraumatic stress disorder. Community Mental Health Journal, 53, 183-201. http://doi.org/10.1007/s10597-016-0047-x

$<$ REFJ $>$ Bryant, R. A. (2007). Acute stress disorder treatment guidelines. International Society Trauma Stress Studies.

$<$ REFJ > Bryant, R. A. (2013). An update of acute stress disorder. PTSD Research Quarterly, 24(1).

$<$ REFJ> Bryant, R. A. (2016). Acute stress disorder: What it is and how to treat it. The Guilford Press.

$<$ REFJ> Bryant, R. A. (2018). The current evidence of acute stress disorder. Current Psychiatry Reports, 20, Article 111. http://doi.org/10.1007/s11920-018-0976-x

$<$ REFJ> Bryant, R. A., Creamer, M., O’Donnell, M., Silove, D., \& McFarlane, A. (2012). The capacity of acute stress disorder to predict posttraumatic psychiatric disorders. Journal of Psychiatric Research, 46, 168-173. http://doi.org/10.1016/j.jpsychires.2011.10.007

$<$ REFJ> Bryant, R. A., Creamer, M., O’Donnell, M., Silove, D., McFarlane, A. C., \& Forbes, D. (2015). A comparison of the capacity of DSM-IV and DSM-V acute stress disorder definitions to predict posttraumatic stress disorder and related disorders. Journal of Clinical Psychiatry, 76(4), 391-397. http://doi.org/10.4088/JCP.13m08731

<REFJ> Bryant, R. A., Friedman, M. J., Spiegel, D., Ursano, R., \& Strain, J. (2010). A review of acute stress disorder in DSM-5. Depression and Anxiety, 28(9), 1-16. http://doi.org/10.1002/da.20737

<REFJ> Bryant, R. A., Harvey, A. G, Dang, S. T., \& Sackville, T. (1998). Assessing acute stress disorder: Psychometric properties of a structured clinical interview. Psychological Assessment, 10, 215-220. http://doi.org/10.1037/1040-3590.10.3.215

$<$ REFJ> Bryant, R. A., Moulds, M. L., \& Guthrie, R. M. (2000). Acute Stress Disorder Scale: A selfreport measure of acute stress disorder. Psychological Assessment, 12(1) 61-68. http://doi.org/10.1037/1040-3590.12.1.61

$<$ REFJ > Campbell, J. \& Herzinger, C. (2010). Statistics and single subject research methodology. In D. L. Gast (Ed.), Single subject research methodology in behavioral sciences (pp. 417-453). Rotledge.

<REFJ > Cohen, J. (1988). Statistical power analysis for the behavioral sciences. Routledge Academic. https://doi.org/10.1016/C2013-0-10517-X

<REFJ> Cova, F., \& Rincón, P. (2010). El terremoto y tsunami del 27-F y sus efectos en la salud mental [The $27-\mathrm{F}$ earthquake and tsunami and their effects on mental health]. Terapia Psicológica, 28(2), 179-185. http://doi.org/10.4067/S0718-48082010000200006 
$<$ REFJ> Cumming, G. (2012). Understanding the new statistics: Effect sizes, confidence intervals, and meta-analysis. Routledge. https://doi.org/10.4324/9780203807002

$<$ REFJ> Durón-Figueroa, R., Cárdenas-López, G., \& De la Rosa, A. (2018). Estructura factorial de la Escala de Trastorno por Estrés Agudo en población mexicana [Factor structure of the Acute Stress Disorder Scale in mexican population]. Journal of Behavior, Health \& Social Issues, 10(2), 29-38. http://doi.org/10.22201/fesi.20070780.2017.9.2.68381

<REFJ> Durón-Figueroa, R., Cárdenas-López, G., Castro, J., \& De la Rosa (2019). Adaptación de la Lista Checable de Trastorno por Estrés Postraumático para DSM-5 en población mexicana. Acta de Investigación Psicológica, 9(1), 26-36.

$<$ REFJ > Echeburúa, E., Corral, P., \& Fernández-Montalvo, J. (2000). Escala de Inadaptación (EI): Propiedades psicométricas en contextos clínicos [Maladjustment Scale: Psychometric properties in clinical contexts]. Análisis y Modificación de la Conducta, 26(107), 325-334.

$<$ REFJ> Foa, E. B., \& Kozak, M. J. (1986). Emotional processing of fear: Exposure to corrective information. Psychological Bulletin, 99, 20-35. http://doi.org/10.1037/0033-2909.99.1.20

$<$ REFJ> Galea, S., Nandi, A., \& Vlahov, D. (2005). The epidemiology of posttraumatic stress disorder after disasters. Epidemiological Reviews, 27, 78-91. http://doi.org/10.1093/epirev/mxi003

$<$ REFJ> Howlett, J. R., \& Stein, M. B. (2016). Prevention of trauma and stressor-related disorders: A review. Neuropsychopharmacology Reviews, 41, 357-369. http://doi.org/10.1038/npp.2015.261

<REFJ> Iraurgi, I. (2010). Evaluación de resultados clínicos (y III): Índices de Cambio Fiable (ICF) como estimadores del cambio clínicamente significativo [Evaluation of clinical results Reliable Change Indices as estimators of clinically significant change]. Norte de Salud Mental, 8(36), 105-122.

$<$ REFJ> Jacobson, N. S., \& Truax, P. (1991). Clinical significance: A statistical approach to defining meaningful change in psychotherapy research. Journal of Consulting and Clinical Psychology, 59(1), 12-19. http://doi.org/10.1037/0022-006X.59.1.12

<REFJ> Jurado, S., Villegas, M., Méndez, L., Rodríguez, F., Loperena, V., \& Varela, R. (1998). La estandarización del Inventario de Depresión de Beck para los residentes de la ciudad de México [Standardizing the Beck Depression Inventory for residents of Mexico City]. Salud Mental, 21(3), 26-31.

$<$ REFJ> Kazdin, A. (2011). Single-case research designs: Methods for clinical and applied settings $\left(2^{\text {nd }} E d.\right)$. Oxford University Press.

$<$ REFJ> Kliem, S., \& Kröger, C. (2013). Prevention of chronic PTSD with early cognitive behavioral therapy. A meta-analysis using mixed-effects modeling. Behavior Research and Therapy, 51(11), 753-761. http://doi.org/10.1016/j.brat.2013.08.005

$<$ REFJ> Medina-Mora, M. E., Borgues, G., Lara, C., Ramos, L., Zambrano, J., \& Fleiz, C. (2005). Prevalencia de sucesos violentos y de trastorno por estrés postraumático en población mexicana [Prevalence of violent events and post-traumatic stress disorder in the Mexican population]. Salud Pública, 47, 8-22. http://doi.org/10.1590/S0036-36342005000100004

$<$ REFJ $>$ McLean, C. P., \& Foa, E. B. (2011). Prolonged exposure therapy for post-traumatic stress disorder: A review of evidence and dissemination. Expert Review of Neurotherapeutics, 11(8), 1151-1163. http://doi.org/10.1586/ern.11.94

$<$ REFJ $>$ Mordeno, I.G. \& Cue, M.P. (2015). Examining the latent structure of acute stress disorder symptoms in Filipino-victims of a flashflood disaster. Community Mental Health Journal, 51, 613-620. http://doi.org/10.1007/s10597-015-9826-z

$<$ REFJ> Neria, Y., Nandi, A., \& Galea, S. (2008). Posttraumatic Stress Disorder following disasters: A systematic review. Psychological Medicine, 38(4), 467-480. http://doi.org/10.1017/S0033291707001353

$<$ REFJ> Norris, F. H., Friedman, M. J., Watson, P. J., Byrne, C. M., Díaz, E., \& Kaniasty, K. (2002) 60,000 disaster victims speak: Part I. An empirical review of the empirical literature, 19812001. Psychiatry: Interpersonal and Biological Processes, 65, 207-239. http://doi.org/10.1521/psyc.65.3.207.20173

$<$ REFJ > Orozco, R., Borges, G., Benjet, C., Medina-Mora, M., \& López-Carrillo, L. (2008). Eventos traumáticos de vida y trastorno de estrés postraumático en adolescentes mexicanos: 
Resultados de encuesta [Traumatic life events and post-traumatic stress disorder in Mexican adolescents: Survey results]. Salud Publica México, 50(1), 29-37.

$<$ REFJ> Robles, R., Varela, R., Jurado S., \& Páez, F. (2001). Versión mexicana del Inventario de Ansiedad de Beck: Propiedades psicométricas [Mexican version of the Beck Anxiety Inventory: Psychometric properties]. Revista Mexicana de Psicología, 18, 211-218.

$<$ REFJ > Sattler, D. N., Glower de Alvarado, A. M., Blandon de Castro, N., Van Male, R., Zetino, A. M., \& Vega, R. (2006). El Salvador earthquakes: Relationships among acute stress disorder symptoms, depression, traumatic event exposure and resource loss. Journal of Traumatic Stress, 19(6), 879-893. http://doi.org/10.1002/jts.20174

$<$ REFJ> Soldatos, C. R., Paparrigopoulos, T. J., Pappa, D. A., \& Christodoulou, G. N. (2006). Early post-traumatic stress disorder in relation to acute stress reaction: An ICD-10 study among help seekers following an earthquake. Psychiatry Research, 143, 245-253. http://doi.org/10.1016/j.psychres.2005.05.018

<REFJ> Tapia, R., Sepúlveda, J., Medina-Mora, M., Caraveo, J., \& De la Fuente, J. (1987). Prevalencia del síndrome de estrés postraumático en población sobreviviente a un desastre natural [Prevalence of post-traumatic stress syndrome in a population surviving a natural disaster]. Salud Pública México, 29(5), 406-411.

<REFJ> Weathers, F., Litz, B., Keane, T., Palmieri, P., Marx, B., \& Schnurr, P. (2013). The PTSD Checklist for DSM-5 (PCL-5). https://www.ptsd.va.gov/professional/assessment/adult$\underline{\mathrm{sr} / \mathrm{ptsd} \text {-checklist.asp }}$

Comentat [S.JP4]: En lo que respecta a los autores que firman sus trabajos con los dos apellidos, se debe reflejar así en la cita y en la referencia, aunque no lleven el guión de apellido compuesto. Por favor verifiquen y modifiquen en consecuencia. 
Table 1.

Clinical Significance and RCI of Each Participant for the Clinical Measures at Postassessment and One, Three and Six-month Follow-ups

\begin{tabular}{|c|c|c|c|c|}
\hline Measure & Post-assessment & $\begin{array}{l}\text { One-month } \\
\text { follow-up }\end{array}$ & $\begin{array}{l}\text { Three- } \\
\text { month } \\
\text { follow-up }\end{array}$ & $\begin{array}{l}\text { Six-month } \\
\text { follow-up }\end{array}$ \\
\hline \multicolumn{5}{|l|}{ PCL-5 } \\
\hline Recovered & $14(100 \%)$ & $14(100 \%)$ & $14(100 \%)$ & $14(100 \%)$ \\
\hline Improved & $0(\%)$ & $0(\%)$ & $0(\%)$ & $0(\%)$ \\
\hline No change & $0(\%)$ & $0(\%)$ & $0(\%)$ & $0(\%)$ \\
\hline Deteriorated & $0(\%)$ & $0(\%)$ & $0(\%)$ & $0(\%)$ \\
\hline \multicolumn{5}{|l|}{ BAI } \\
\hline Recovered & $7(50 \%)$ & $10(71.4 \%)$ & $9(64.2 \%)$ & $13(92.8)$ \\
\hline Improved & $4(28.5 \%)$ & $4(28.5 \%)$ & $4(28.5 \%)$ & $1(7.1 \%)$ \\
\hline No change & $3(21.4 \%)$ & $0(\%)$ & $1(7.1 \%)$ & $0(\%)$ \\
\hline Deteriorated & $0(\%)$ & $0(\%)$ & $0(\%)$ & $0(\%)$ \\
\hline \multicolumn{5}{|l|}{ BDI } \\
\hline Recovered & $8(57.1 \%)$ & $11(78.5 \%)$ & $10(71.4 \%)$ & $11(78.5 \%)$ \\
\hline Improved & $5(35.7 \%)$ & $2(14.2)$ & $3(21.4 \%)$ & $3(21.4 \%)$ \\
\hline No change & $1(7.1 \%)$ & $1(7.1 \%)$ & $1(7.1 \%)$ & $0(\%)$ \\
\hline Deteriorated & $0(\%)$ & $0(\%)$ & $0(\%)$ & $0(\%)$ \\
\hline \multicolumn{5}{|l|}{ MS } \\
\hline Recovered & $14(100 \%)$ & $14(100 \%)$ & $14(100 \%)$ & $14(100 \%)$ \\
\hline Improved & $0(\%)$ & $0(\%)$ & $0(\%)$ & $0(\%)$ \\
\hline No change & $0(\%)$ & $0(\%)$ & $0(\%)$ & $0(\%)$ \\
\hline Deteriorated & $0(\%)$ & $0(\%)$ & $0(\%)$ & $0(\%)$ \\
\hline
\end{tabular}

Note. PCL-5 = PTSD Checklist for DSM-5; BAI = Beck Anxiety Inventory; BDI = Beck

Depression Inventory; MS = Maladjustment Scale. 
Table 2.

Intra-group Comparisons between Pre and Post-assessment and One, Three and Six-month Follow-ups

\begin{tabular}{|c|c|c|c|c|c|c|c|c|c|c|c|c|}
\hline \multirow[b]{2}{*}{ Measures } & \multicolumn{3}{|c|}{ Post-assessment } & \multicolumn{3}{|c|}{ One-month follow-up } & \multicolumn{3}{|c|}{ Three-month follow-up } & \multicolumn{3}{|c|}{ Six-month follow-up } \\
\hline & $M(S D)$ & $Z$ & $g$ & $M(S D)$ & $Z$ & $g$ & $M(S D)$ & $Z$ & $g$ & $M(S D)$ & Z & $g$ \\
\hline PCL-5 & $8.5(5.2)$ & $-3.297 * *$ & 3.7 & $5.1(3.3)$ & $-3.297 * *$ & 4.0 & $6.0(3.8)$ & $-3.297 * *$ & 3.9 & $4.6(2.5)$ & $-3.303 * *$ & 4.1 \\
\hline BAI & $7.2(4.3)$ & $-3.300^{* *}$ & 1.8 & $5.1(3.3)$ & $-3.297 * *$ & 2.0 & $5.3(3.0)$ & $-3.300 * *$ & 2.0 & $4.5(2.7)$ & $-3.300^{* *}$ & 2.0 \\
\hline BDI & $5.0(3.4)$ & $-3.304 * *$ & 1.9 & $3.5(3.3)$ & $-3.297 * *$ & 2.1 & $3.5(3.1)$ & $-3.299 * *$ & 2.1 & $2.8(3.0)$ & $-3.298 * *$ & 2.0 \\
\hline MS & $2.7(1.8)$ & $-3.306^{* *}$ & 2.9 & $1.4(1.7)$ & $-3.300^{* *}$ & 3.2 & $1.3(1.7)$ & $-3.309 * *$ & 3.2 & $1.7(1.7)$ & $-3.300 * *$ & 3.1 \\
\hline
\end{tabular}

Note. $\mathrm{Z}=$ Comparison to pre-assessment of the Wilcoxon rank-sum test; $g=$ Cohen's $d$ effect size corrected with Hedge's $g$ for small samples; PCL-5 = PTSD Checklist for DSM-5; BAI = Beck Anxiety Inventory; BDI = Beck Depression Inventory; MS = Maladjustment Scale.

$* p<.000 . * * \mathrm{p}<.001$ 
$<\mathrm{H} 1>$ Appendix. Clinical Measures Graphs for Visual Inspection

$<\mathrm{H} 2>$ PTSD Clinical Measures (PCL-5)
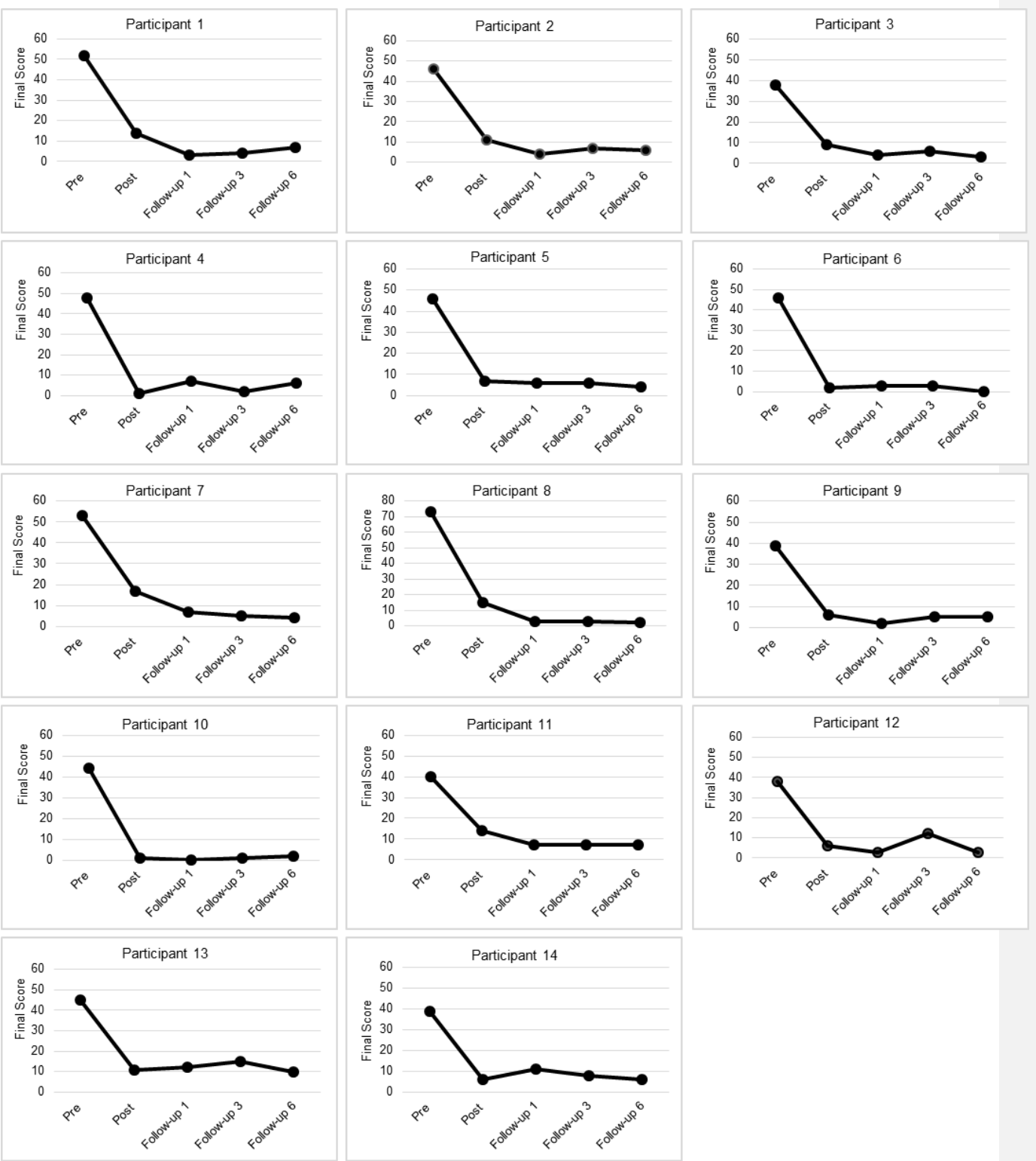
$<\mathrm{H} 2>$ Anxiety Clinical Measures (BAI)
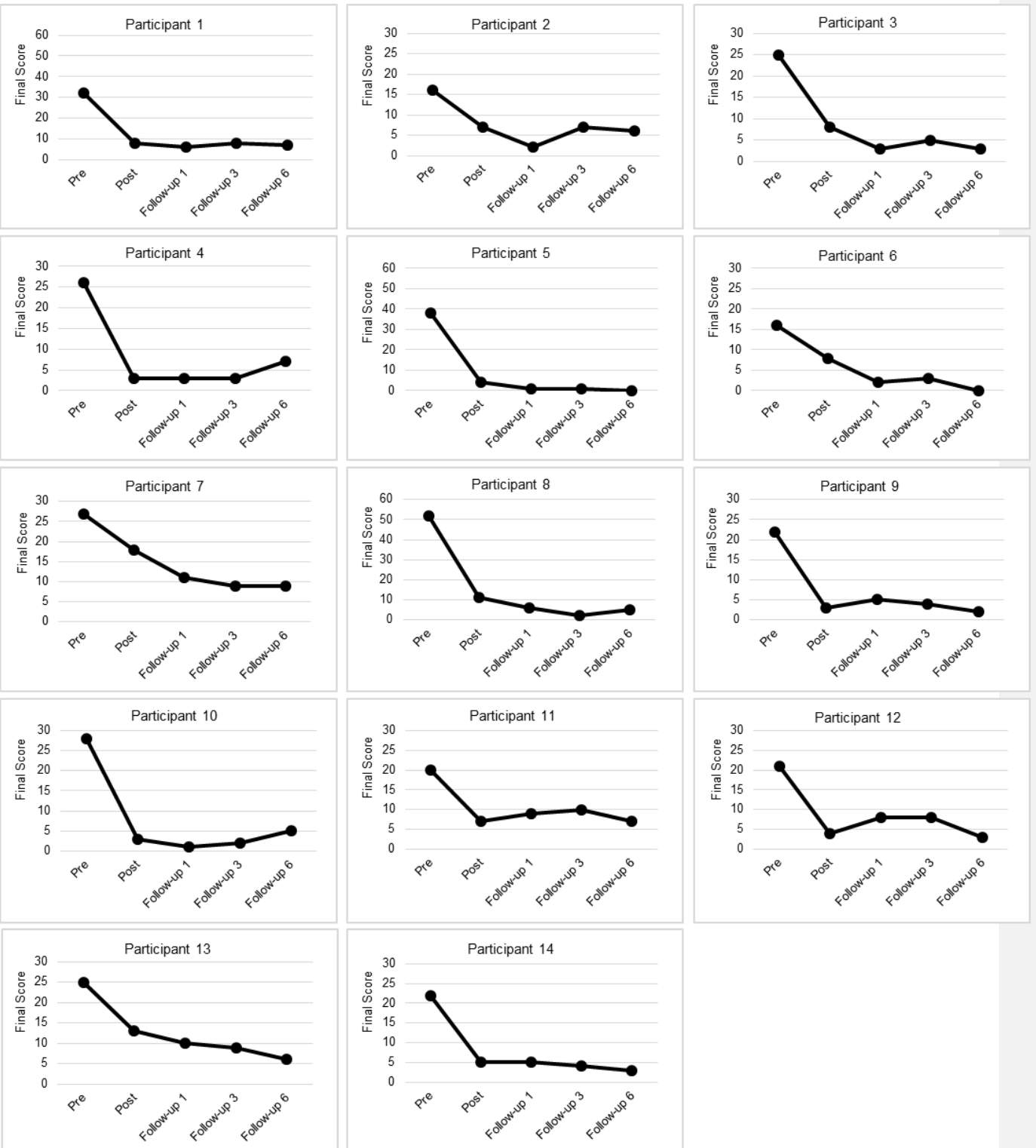
$<\mathrm{H} 2>$ Depression Clinical Measures (BDI)
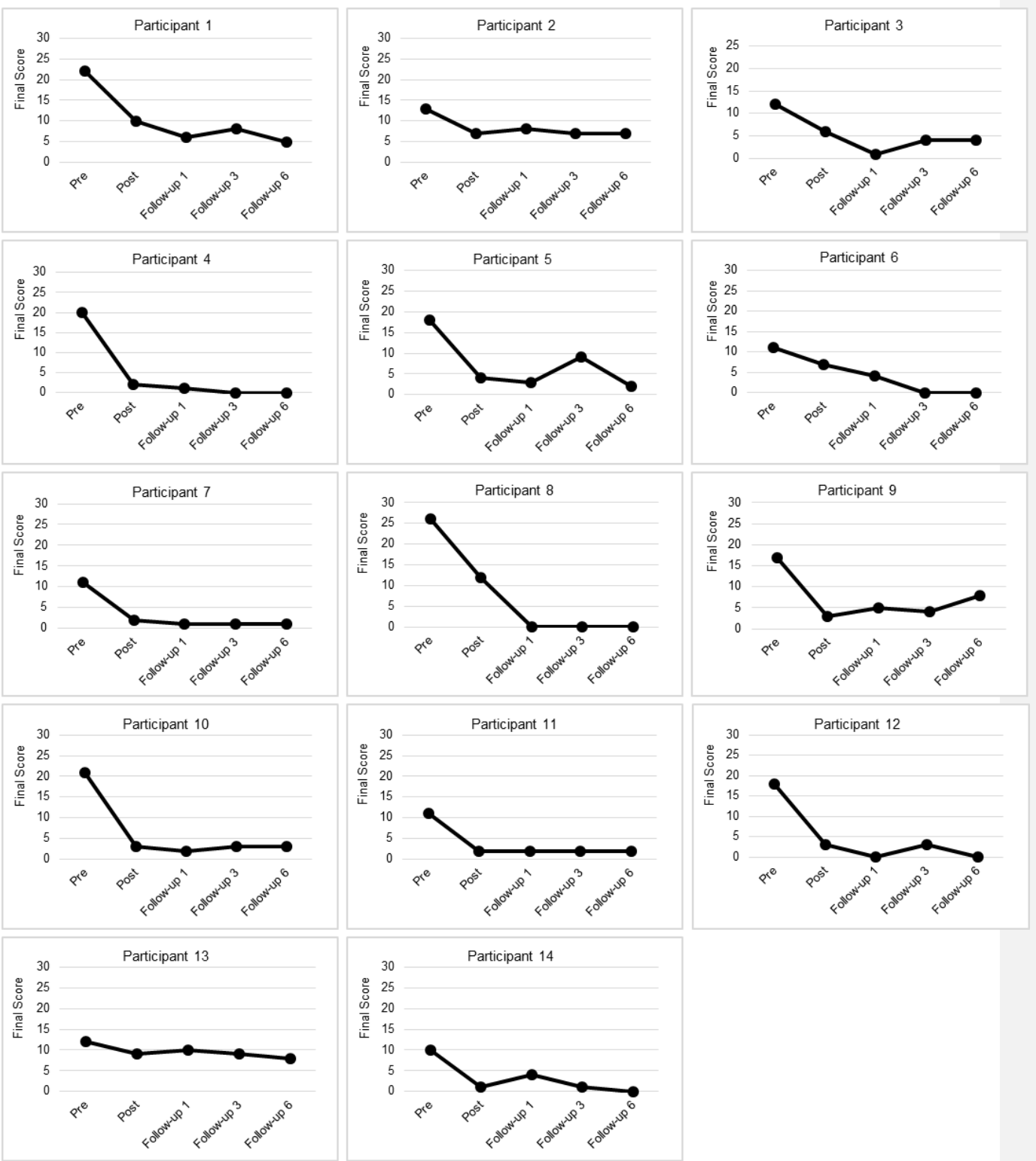
$<\mathrm{H} 2>$ Maladjustment Clinical Measures (MS)
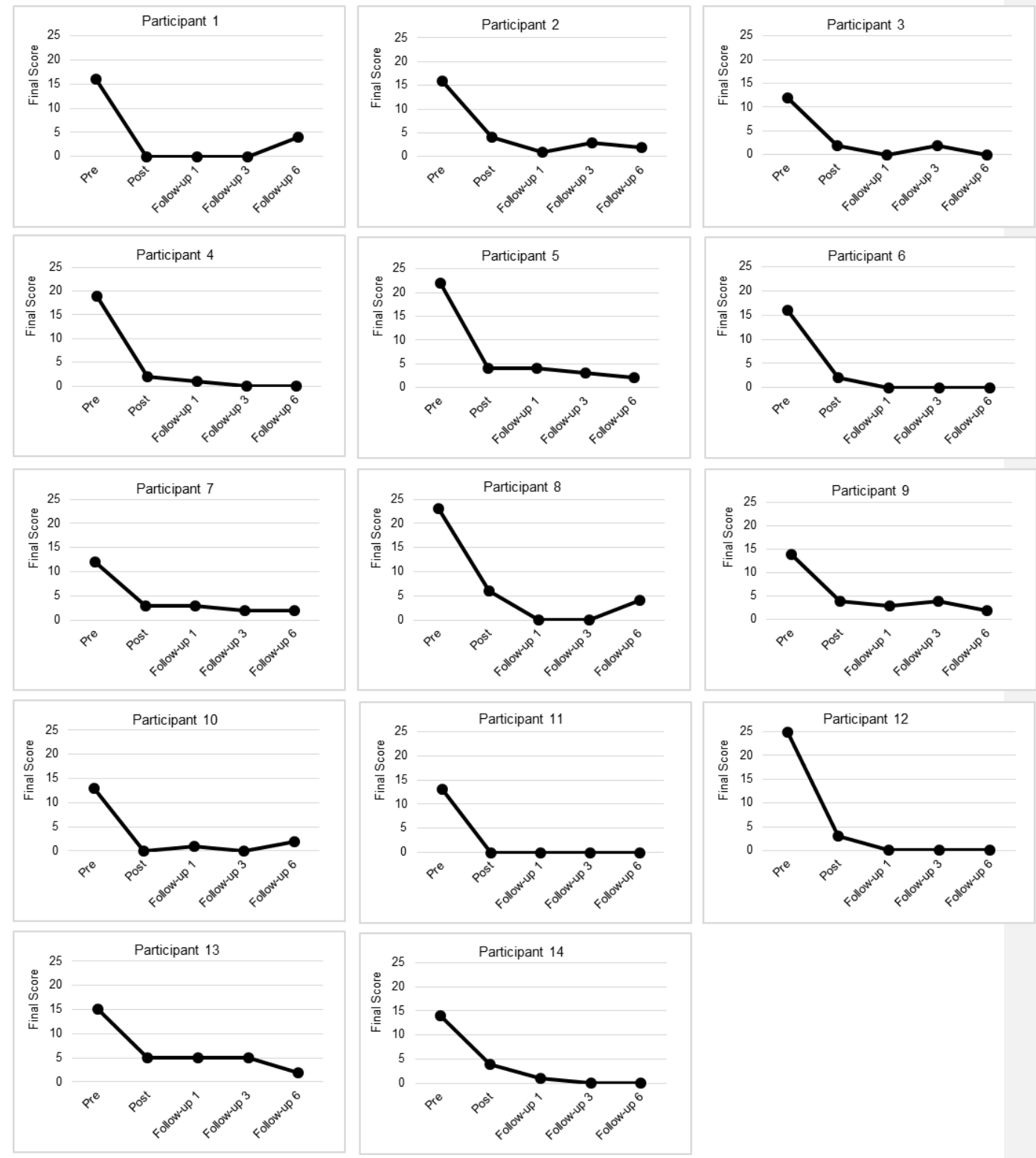

24 